\title{
Development of tivanisiran, a topical siRNA designed to treat dry eye disease
}

\author{
Anne-Marie Bleau*, Verónica Ruz, Victoria González, Tamara Martínez, Beatriz Vargas and Ana Isabel Jimenez \\ Sylentis, Madrid, Spain
}

\section{Introduction}

Dry eye is disease (DED) emerges as a new prevalent ocular affectation in modern-day, affecting between 5 to $50 \%$ of the population worldwide [1]. DED is defined a multifactorial disease of the ocular surface that combines alterations of the tear film, inflammatory processes and neurosensory abnormalities [2]. While the main sign of DED is the appearance of corneal damage, symptoms generally manifest as ocular pain/discomfort, dryness, itching and burning. Management of DED primarily relies on artificial tear substitute use, although other strategies such as autologous serum, punctal occlusion, lid hygiene and dietary changes are common [3]. In the United States, only two prescription drugs have been approved for the treatment of DED: a cyclosporine ophthalmic emulsion (also approved in Europe) and lifitegrast $[4,5]$. These non-steroidal anti-inflammatory agents only present efficacy in a subset of patients. There is thus a significant unmet need for effective treatments that will improve signs and symptoms of DED.

\section{Tivanisiran's mechanism of action}

Sylentis is developing tivanisiran (former SYL1001), a novel small interfering RNA (siRNA) aimed at treating DED. Tivanisiran is a non-modified, 19-base pair, oligonucleotide sodium salt specifically designed to silence the Transient Receptor Potential Cation channel subfamily V member 1 (TRPV1) mRNA. Transient silencing occurs via the RNA interference (RNAi) mechanism of action in which the siRNA is required to reach the cytoplasm where it can bind to complementary TRPV1 mRNA [6,7]. This produces a degradation of the mRNA, preventing its translation into protein. TRPV1 is expressed in primary afferent nociceptive neurons of the peripheral nervous system, in the central nervous system and in non-neuronal tissues such as the skin, epithelial airway and different eye tissues $[8,9]$. The cornea is densely innervated by sensory afferent fibers of the trigeminal nerve, where TRPV1 acts as nociceptive transducer for the sensing and transmission of pain stimuli [10]. In corneal epithelial cells, the receptor can be implicated in inflammatory and fibrogenesis processes, as well as regulation of response to stress [11]. Since none of the commercially available treatment target the pain pathway, TRPV1 appears as an attractive and innovative target for the treatment of this disease.

\section{In vitro and in vivo studies for tivanisiran}

Non-clinical studies of tivanisiran have been previously described in more details [12]. Briefly, in vitro transfection of tivanisiran was shown to produce a 50-60\% decrease in TRPV1 mRNA levels. High specificity was reported: the compound acts specifically on TRPV1 rather than other members of the TRPV family or other sequence-related receptors, minimizing the risk of toxicity. Corroborating this observation, tivanisiran did not affect cell viability. Interestingly, biodistribution studies after single topical instillation of tivanisiran in New Zealand rabbits demonstrated that the compound efficiently reached corneal and conjunctival tissues five and $30 \mathrm{~min}$ after administration. Of note, intracellular siRNAs are rapidly phosphorylated on their $5^{\prime} \mathrm{P}$, allowing differential quantification of the compound (phosphorylated and non-phosphorylated forms). In these studies, the highest amount of tivanisiran was detected in response to the dose of $1.125 \%$.

Non-clinical pharmacokinetic studies were conducted in two species after topical administration of tivanisiran (at doses up to six times the clinical dose) during 28 consecutive days. Analysis revealed that the compound was below the Lower Limit of Quantification (LLOQ) at all tested time-points and in response to all dose-levels in all plasma and systemic tissues, suggesting minimal systemic absorption. In these animals, tivanisiran did not produce any apparent alterations in clinical signs or ophthalmologic exams. Most importantly, corneal sensitivity, as assessed by esthesiometer, confirmed that the compound is devoid of anesthetic effect. Overall, these observations indicate that tivanisiran displays a good local and systemic tolerability.

\section{Clinical studies for tivanisiran}

Safety and efficacy of tivanisiran have been evaluated in three clinical trials [13]. Tivanisiran sodium salt ophthalmic solution was formulated in absence of preservative at $\mathrm{pH} 7.2$ at five nominal concentrations: 3.75 $\mathrm{mg} / \mathrm{mL}$ (0.375\%), $7.5 \mathrm{mg} / \mathrm{mL}$ (0.75\%), $11.25 \mathrm{mg} / \mathrm{mL}$ (1.125\%), $15 \mathrm{mg} /$ $\mathrm{mL}(1.5 \%)$ and $22.5 \mathrm{mg} / \mathrm{mL}$ (2.25\%). In the phase I study (EudraCT No: 2010-023113-56, NCT01438281), thirty healthy volunteers received single or multiple administrations of tivanisiran for seven consecutive days, with the counter-lateral eye used as control. No related adverse events (AEs) were reported and no clinically significant changes were observed for ocular and physical examinations. Tivanisiran could not be detected in any of the plasma samples collected regardless of the time-points (LLOQ $<10 \mathrm{ng} / \mathrm{mL}$ ).

Thereafter, two phase II trials, named SYL1001_II and SYL1001_III (EudraCT No: 2012-001177-93, NCT01776658 and EudraCT No 2014004857-15, NCT02455999) were conducted in a total of 126 patients with mild to moderate DED [13]. Each trial tested two different doses of tivanisiran that were compared with placebo (approximately 20 patients per arm). Tivanisiran was instilled once a day during 10 consecutive

${ }^{\star}$ Correspondence to: Anne-Marie Bleau, Sylentis, PCM-c/Santiago Grisolía 2, 28760 Madrid, Spain, Tel: +3491 8043817; Fax: +3491 8049597; E-mail: ambleau@sylentis. com

Received: May 15, 2018; Accepted: June 08, 2018; Published: June 13, 2018 
days. The trials met the primary endpoint of identifying the most efficient dose in reducing signs and symptoms of DED. Tivanisiran $1.125 \%$ decreased the scoring on the visual analogue scale (VAS) for ocular pain as compared with placebo. Differences were statistically significant from Day 4 and were maintained until the end of treatment. Following this short time treatment-period, no changes were detected in the Ocular Surface Disease Index (OSDI). Signs of DED were also affected in these trials. A significant percentage of patients showed improvement in conjunctival hyperemia after 10 days of instillation with tivanisiran $1.125 \%$ and $0.75 \%$ compared with placebo. Similar findings were made for corneal fluorescein staining (CFS), although difference did not reach statistical significance. Tear break up time (TBUT) was prolonged of two seconds at Day 10 of treatment with tivanisiran $1.125 \%$ respective to baseline. As previously reported, the incidence of $\mathrm{AE}$ was similar between tivanisiran-treated groups and placebo. Overall, these trials suggest a good safety profile for tivansiran and the dose of $1.125 \%$ was selected for future trials.

\section{Current trial for tivanisiran}

HELIX study (EudraCT No: 2016-003903-79, NCT03108664) is ongoing to assess the effect of tivanisiran $1.125 \%$ in 300 patients with moderate to severe DED. The trial is being conducted in six different countries, involving more than 39 sites. Primary endpoints are change from baseline in VAS for ocular discomfort/pain, CFS and conjunctival hyperemia. Confirmation of efficacy will allow to determine whether tivanisiran would be beneficial for these patients and may satisfy the unmet needs of DED.

\section{Conflict of Interest}

AMB, VR, VG TM, BV and AIJ are employed by Sylentis.

\section{References}

1. Stapleton F, Alves M, Bunya VY, Jalbert I, Lekhanont K, et al. (2017) TFOS DEWS II Epidemiology Report. Ocul Surf 15: 334-365. [Crossref]

2. Craig JP, Nichols KK, Akpek EK, Caffery B, Dua HS, et al. (2017) TFOS DEWS II Definition and Classification Report. Ocul Surf 15: 276-283. [Crossref]

3. Jones L, Downie LE, Korb D, Benitez-Del-Castillo JM, Dana R, et al. (2017) TFOS DEWS II Management and Therapy Report. Ocul Surf 15: 575-628. [Crossref]

4. Leonardi A, Van Setten G, Amrane M, Ismail D, Garrigue JS, et al. (2016) Efficacy and safety of $0.1 \%$ cyclosporine A cationic emulsion in the treatment of severe dry eye disease: a multicenter randomized trial. Eur J Ophthalmol 26: 287-296. [Crossref]

5. Schultz C (2014) Safety and efficacy of cyclosporine in the treatment of chronic dry eye. Ophthalmol Eye Dis 6: 37-42. [Crossref]

6. Lu PY, Xie F, Woodle MC (2005) In vivo application of RNA interference: from functional genomics to therapeutics. Adv Genet 54: 117-142. [Crossref]

7. Zimmermann TS, Lee AC, Akinc A, Bramlage B, Bumcrot D, et al. (2006) RNAimediated gene silencing in non-human primates. Nature 441: 111-114. [Crossref]

8. Zan Pan, J.E.C.-A.a.P.S.R. (2012) Transient Receptor Potential (TRP) Channels in the Eye. In: Advances in Ophthalmology. Rumelt S (Ed.) InTech. p. 35-46.

9. Martínez-García MC, Martínez T, Pañeda C, Gallego P, Jimenez AI, et al. (2013) Differential expression and localization of transient receptor potential vanilloid 1 in rabbit and human eyes. Histol Histopathol 28: p. 1507-1516. [Crossref]

10. Szallasi A, Cortright DN, Blum CA, Eid SR (2007) The vanilloid receptor TRPV1: 10 years from channel cloning to antagonist proof-of-concept. Nat Rev Drug Discov 6: 357-372. [Crossref]

11. Pan Z, Wang Z, Yang H, Zhang F, Reinach PS (2011) TRPV1 activation is required for hypertonicity-stimulated inflammatory cytokine release in human corneal epithelial cells. Invest Ophthalmol Vis Sci 52: p. 485-493. [Crossref]

12. Moreno-Montañés J, Bleau AM, Jimenez AI (2018) Tivanisiran, a novel siRNA for the treatment of dry eye disease. Expert Opin Investig Drugs 27: 421-426. [Crossref]

13. Benitez-Del-Castillo JM, Moreno-Montañés J, Jiménez-Alfaro I, Muñoz-Negrete FJ, Turman K, et al. (2016) Safety and Efficacy Clinical Trials for SYL1001, a Novel Short Interfering RNA for the Treatment of Dry Eye Disease. Invest Ophthalmol Vis Sci 57: 6447-6454. [Crossref]

Copyright: (C2018 Bleau A. This is an open-access article distributed under the terms of the Creative Commons Attribution License, which permits unrestricted use, distribution, and reproduction in any medium, provided the original author and source are credited. 